\title{
La Polynésie des vahinés et la nature des femmes : une utopie occidentale masculine
}

\section{Serge Tcherkezoff}

\section{(2) OpenEdition}

\section{Journals}

\section{Édition électronique}

URL : https://journals.openedition.org/clio/1742

DOI : 10.4000/clio.1742

ISSN : 1777-5299

Éditeur

Belin

Édition imprimée

Date de publication : 1 novembre 2005

Pagination : 63-82

ISBN : 2-85816-821-0

ISSN : $1252-7017$

\section{Référence électronique}

Serge Tcherkezoff, «La Polynésie des vahinés et la nature des femmes : une utopie occidentale masculine », Clio. Histoire, femmes et sociétés [En ligne], 22 | 2005, mis en ligne le 01 décembre 2007, consulté le 22 avril 2022. URL : http://journals.openedition.org/clio/1742 ; DOI : https://doi.org/ $10.4000 /$ clio. 1742

Ce document a été généré automatiquement le 22 avril 2022.

Tous droits réservés 


\title{
La Polynésie des vahinés et la nature des femmes : une utopie occidentale masculine
}

\author{
Serge Tcherkezoff
}

\section{NOTE DE L'ÉDITEUR}

Ce texte fut élaboré au Gender Relations Center (ANU), début 2005, lorsque l'auteur était accueilli comme ARC Fellow. L'auteur remercie le GRC pour son accueil et pour toute l'aide matérielle apportée.

Les premiers contacts et la surinterprétation française

1 En 1771, le Voyage autour du monde de Bougainville fait connaître la «NouvelleCythère » (Tahiti). Pourquoi ce nom ? Avant même de débarquer, les Français reçurent une visite à bord: une «jeune fille » se présenta, «laissa tomber négligemment un pagne qui la couvrait, et parut aux yeux de tous telle que Vénus se fit voir au berger phrygien : elle en avait la forme céleste ». Bougainville donna l'ordre à ses marins de ne pas bouger et les visiteurs tahitiens s'en allèrent. Mais quelles avaient été les intentions de cette jeune fille? Bougainville ne laissa aucun doute à son lecteur, quand, quelques pages plus loin, mentionnant les journées qui suivirent, il affirma :

Chaque jour nos gens se promenaient dans le pays [...]. On les invitait à entrer dans les maisons, on leur y donnait à manger; mais ce n'est pas à une collation légère que se borne ici la civilité des maîtres de maison; ils leur offraient des jeunes filles. La terre se jonchait de feuillage et de fleurs, et des musiciens chantaient aux accords de la flûte un hymne de jouissance. Vénus est ici la déesse de l'hospitalité, son culte n'y admet point de mystères, et chaque jouissance est une fête pour la nation ${ }^{1}$.

2 Les Français ne restèrent que dix jours (avril 1768), et ne comprenaient pas un mot de la langue locale 2 . Mais leur opinion fut unanime : c'était un « hymne de jouissance » que les Tahitiens chantaient. Le naturaliste du voyage, Philibert Commerson, et les autres 
compagnons, tels le volontaire Fesche, le Prince de Nassau, le chirurgien Vivès, etc., tinrent les mêmes propos ${ }^{3}$. Tous considérèrent que la réception fut une "hospitalité " sexuelle et notèrent que cette sexualité tahitienne réclamait d'être pratiquée en public. Ils estimèrent aussi que ce devait être une coutume locale: c'est ainsi que se pratiquerait l'amour entre les Tahitiens. Les Français n'imaginèrent pas un instant que les Tahitiens auraient pu organiser une réception inhabituelle, pour des êtres extraordinaires. Ils crurent être reçus comme des "amis» ou des «alliés »". Tous remarquèrent que les femmes qui semblaient mariées ne s'offraient pas et que les présentations sexuelles étaient limitées aux «jeunes filles». Mais au lieu de s'interroger davantage sur cette étrange restriction, ils en conclurent immédiatement que la coutume générale était la liberté sexuelle, et que seule la jalousie d'un mari y mettait un frein. Nassau sembla voir que les femmes étaient « rigidement assujettis [sic] aux devoirs conjugaux» tandis que les filles, «maîtresses d'elle-même, disposent de leur charme à leur bon plaisirs $[s i c]^{5} »$. Commerson écrivit les mêmes mots ${ }^{6}$.

3 En 1769, James Cook fit escale à Tahiti. Quand son récit parut en 1773 tous les lecteurs retinrent une scène, décrite dans le style d'un Ovide (l'Amirauté britannique avait confié le journal de Cook à un écrivain londonien afin d'obtenir un récit digne d'une œuvre littéraire, mais cet écrivain déforma le contenu original ${ }^{7}$ ). Devant le campement anglais établi sur la plage :

Un jeune homme, haut de près de six pieds, exécuta les rites de Vénus avec une petite fille de onze ou douze ans, devant plusieurs de nos gens et un grand nombre d'indigènes, sans avoir le moins du monde l'impression de faire quelque chose d'indécent ou de déplacé, mais, semble-t-il, avec le sentiment de se conformer aux usages du lieu.

4 En fait, Cook avait simplement noté dans son journal que les dignitaires tahitiens firent s'allonger les deux jeunes gens, et ne précisa pas si l'acte sexuel eut lieu. Un autre membre de l'expédition raconta plus tard que rien ne se passa, car les jeunes gens étaient terrorisés ${ }^{8}$. Mais, à l'époque, aucun lecteur ne put l'apprendre et tous n'eurent à lire que les lignes réécrites par le scribe officiel: les « rites de Vénus furent exécutés » par ces jeunes gens, et en public. Personne, non plus, ne s'interrogea sur le fait que la partenaire féminine du " jeune homme » était une " petite fille de onze ou douze ans » (dans son journal Cook avait même écrit " petite fille de dix ou douze ans ") $)^{9}$. Chacun conclut que, à Tahiti, l'amour se faisait dès que la maturité du corps adolescent le permettait et qu'il se faisait « en public ».

5 Il n'y avait plus aucun doute, conclut Voltaire en $1775^{10}$. Les affirmations de Bougainville étaient difficiles à croire et, en 1771, Voltaire ne fut pas convaincu. Mais,

Quand le Français et l'Anglais sont d'accord, il est démontré qu'ils ne nous ont pas trompé. Je suis encore de l'île de Taïti, j'y admire [... leur] service divin [qui] consiste à faire coucher ensemble un jeune homme et une jeune fille tout nus, en présence de Sa Majesté et de cinq cents courtisans et courtisanes. On peut assurer que les habitants de Taïti ont conservé dans toute sa pureté la plus ancienne religion de la terre ${ }^{11}$.

6 Telle fut en résumé l'histoire de la création d'une opinion commune européenne sur la coutume tahitienne, généralisée ensuite à toutes les activités - en particulier les danses - et à tous les archipels : bref, la « culture polynésienne » en général, la Polynésie des vahinés (mot local pour « femmes »), la Polynésie de la liberté sexuelle.

La mise en scène polynésienne des présentations sexuelles féminines 
7 Nous pouvons savoir ce qui s'est réellement passé par les journaux de bord, en particulier concernant la première arrivée française à Tahiti (Bougainville : avril 1768) et la première arrivée française à Samoa (Lapérouse : décembre 1787). Après un examen comparatif détaillé de ces deux cas et de quelques autres, une autre scène apparaît ${ }^{12}$.

Le volontaire Felix Fesche avait noté à Tahiti :

[...] ce qui est arrivé peut-être aux deux tiers des Français : des pères et des mères amenaient leurs filles, engageaient [les Français] à consommer l'œuvre de mariage avec elle. La fille [...] se couchait par terre et se dépouillait de ses vêtements. Plusieurs faisaient des façons quand il s'agissait d'en venir au fait, se laissaient vaincre cependant. Durant l'opération, les assistants insulaires, toujours en grand nombre, faisaient un cercle à l'entour, une branche de feuillage à la main. [...] L'opération finie, la fille pleurait, se consolait aisément ${ }^{13}$.

9 Quelques lignes plus haut, Fesche nous indique que lors de la toute première rencontre, la victime était :

une jeune fille de 12 à 13 ans... On nous invite à manger... Nous voyons ensuite chacun d'eux s'emparer d'une branche de feuillage et se ranger en cercle autour de nous... on apporta une natte que l'on étendit sur la place et sur laquelle s'assid la jeune fille. Les signes de tous les Indiens nous firent bien comprendre le fait dont il s'agissoit... la toile [l'habit] fut bientôt ôtée par la fille elle-même que nous vîmes alors avec le seul habillement que portoit Eve avant son péché... Elle s'étendit sur la natte, frappa sur la poitrine de celui qui étoit l'agresseur, lui faisant entendre qu'elle se donnoit à lui et écarta les deux obstacles qui empêchent l'entrée de ce temple où tant d'hommes sacrifient tous les jours.

10 La conclusion va lancer le mythe de l'amour en public. Devant la jeune fille offerte, le Français qui s'était avancé (le Prince de Nassau) hésite. Mais, parce qu'il y avait «la présence de 50 indiens qui l'environnoient ", il devint trop embarrassé pour aller plus loin. Et Fesche de commenter: "il est bien difficile de surmonter tout d'un coup les idées avec lesquelles on a été nourri... Nous nous cachons pour faire une œuvre aussi naturelle : il la font en public et souvent $\aleph^{14}$.

11 Pour Fesche, il n'y a pas de doute : il s'agit bien de désir sexuel, la seule particularité qu'il croit comprendre - et qui le ravit - est que cette sexualité se pratique « en public et souvent".

12 Nassau commente de la même façon:

les préjugés européens exigent plus de mystère... Heureuse nation qui ne connoit pas les noms odieux de honte et de scandale... pourquoy la reproduction de la plus belle des choses créées ne sera tel pas aussy [sic] une fête publique ? ${ }^{15}$

13 En 1787, l'expédition de Lapérouse fut la première expédition européenne à mettre pied sur le sol samoan. Sur le sujet des rencontres sexuelles, Lapérouse mentionna que seulement de «très jeunes filles » furent impliquées :

l'autel fut dressé dans la case du village la plus apparente, toutes les jalousies furent baissées, les curieux écartés ; la victime fut placée entre les bras d'un vieillard, qui lexortoit à moderer sa douleur, car elle pleuroit; les matrones chantoient et hurloient pendant la cérémonie, et le sacrifice fut consommé en presence des femmes et du vieillard qui servoit d'autel et de prêtre. Toutes les femmes et enfants du vilage étaient au tour et en dehors de la maison soulevant legerement les jalousies et cherchant les plus petites ouvertures entre les nattes pour jouir de ce spectacle $^{16}$.

14 Il y a de quoi s'interroger sur ces scènes organisées par les Polynésiens. Aujourd'hui, les hypothèses qu'on peut avancer sont les suivantes. Tout d'abord, les Polynésiens 
virent dans les Européens des envoyés du monde divin: non des dieux, mais des envoyés de ces dieux, porteurs des mêmes pouvoirs sacrés, mais avec un corps humain. Ils tentèrent d'en capter l'essence en leur présentant des jeunes filles afin que celles-ci tombent enceintes. Pourquoi celles-ci devaient-elles être très jeunes? Suivant une théorie locale de la conception, seule la fille (d'une famille de haut rang) qui n'avait pas encore enfanté avait toutes les chances de capter les pouvoirs divins et de mettre au monde un "enfant sacré ». Dans la théorie tahitienne exposée au Capitaine Bligh en 1789, à l'issue de la cérémonie de mariage, c'est le « dieu » qui vient visiter l'épouse, le mari étant à ses côtés. Le premier enfant est « celui du dieu ».

15 Tout cet ensemble de comportements fut observé dans les pratiques locales par les premiers visiteurs de longue durée, présents à partir de 1790-1830 (aventuriers, missionnaires) : les familles tentaient de marier leurs filles jeunes et vierges aux plus grands chefs. Mais ces observateurs n'en comprenaient pas les raisons. Dans notre hypothèse, il s'agissait de capter les pouvoirs divins à l'origine du titre de chef que cet homme détenait. Pour cela, il fallait que la jeune épouse fût vierge. Ainsi, quand les premiers visiteurs européens arrivèrent, ils furent considérés en partie comme des chefs sacrés ${ }^{17}$. Le même schéma leur fut appliqué.

16 Il y avait donc de quoi s'interroger. Mais nos voyageurs du XVIII siècle se limitèrent à l'idée d'une hospitalité sexuelle dans une société de l'amour libre. De sa visite à Samoa, Lapérouse ne retint qu'une chose: «les jeunes filles avant d'être mariées sont maitresses de leurs faveurs... il est plus vraisemblable qu'en se mariant elles n'ont aucun compte à rendre de leur conduite passée $\aleph^{18}$. Les Français arrivés à Tahiti n'avaient pas été moins aveugles. De retour en France, ils ne parlèrent que de liberté sexuelle et de désir amoureux, dépeignant un Jardin d'Eden où toutes les "femmes ", telles «Ève avant son péché», ne songeaient qu'à l'amour. Un de ceux qui fréquentaient la cour à cette époque écrivit :

M. de Bougainville prétend que dans le pays [...] hommes et femmes se livrent sans pudeur au péché de la chair ; qu'à la face du ciel et de la terre, ils se copulent sur la première natte offerte $[. . .]^{19}$

17 Cette vue, ramassée dans les publications officielles, alimenta ensuite, par le jeu de courtes citations, le savoir qu'en prirent les salons philosophiques, puis l'ensemble du monde académique, jusqu'aux enseignements universitaires contemporains. Au début $\mathrm{du} \mathrm{xx}^{\mathrm{e}}$ siècle, les premières enquêtes de terrain en Polynésie qui se voulaient professionnelles, furent lourdement obérées par cette vision, dans l'anthropologie américaine en tout cas: dans les années 1920, Edward Handy décrivant la jeunesse marquisienne, ou écrivant un traité sur la religion "polynésienne traditionnelle », ou encore Margaret Mead décrivant la jeunesse samoane, tous interprétèrent systématiquement leurs observations de terrain dans le sens d'une Polynésie de l'amour-libre. En retournant de près à ces « observations » et à la manière d'en rendre compte, on peut voir en détail l'étendue de la surinterpré-tation ${ }^{20}$.

18 Mais comment comprendre cet aveuglement initial qui entraîna toutes ces illusions? Pourquoi, devant des scènes de jeunes filles amenées de force, Bougainville et Lapérouse conclurent au règne de Vénus et d'Eros, et à une éducation qui laissait les jeunes femmes "maîtresses de leurs faveurs »? La réponse tient en une phrase, écrite par Bougainville, précisément à propos des jeunes Tahitiennes: «les femmes paraissent ne pas vouloir ce qu'elles désirent le plus ».

La vision européenne masculine : la nature universelle des femmes 
19 La femme est faite pour l'amour. Si elle se dénude en public, c'est pour mieux le faire savoir. Si, en se dénudant, elle montre quelque embarras, c'est que,

[...] soit que la nature ait partout embelli le sexe d'une timidité ingénue, soit que même dans un pays où règne encore la franchise de l'âge d'or, les femmes paraissent ne pas vouloir ce qu'elles désirent le plus ${ }^{21}$.

20 C'est ainsi que Bougainville commenta le fait que la première jeune fille montée à bord ("Vénus ») avait semblé montrer quelque hésitation à se déshabiller (on sait en outre, par les journaux de compagnons, que cette fille était entourée d'adultes tahitiens et que ceux-ci lui donnaient des ordres et ôtèrent ses vêtements).

21 En commentant cette rencontre, Fesche ne vit dans tout cela qu'une chose : «l'envie » des jeunes Tahitiennes «d'offrir avec un de nous, quel qu'il fût, un sacrifice à Vénus " ${ }^{22}$. La formule est remarquable. Tout d'abord, le Français attribue aux Tahitiennes une pulsion «naturelle » qui voudrait que tout individu du sexe mâle soit bon à prendre : " quel qu'il fût... ». D'autre part, Fesche confond en une même divinité Vénus et Amour (Eros). La beauté de la femme, que symbolise la déesse Vénus, n'a donc de sens à ses yeux que lorsqu'elle est « sacrifiée » sur l'autel de l'Amour.

22 Commerson disait des Tahitiens : « ils ne connaissent d'autre dieu que l'Amour. Tous les jours lui sont consacrés, toute l'île est son temple, toutes les femmes en sont les autels, tous les hommes les sacrificateurs ${ }^{23}$.

23 Fesche, à qui nous devons pourtant de savoir ce que furent réellement les présentations sexuelles, nous parle du bonheur de vivre à Tahiti, et n'en parle qu'au masculin :

Si le bonheur consiste dans l'abondance de toutes les choses nécessaires à la vie, à habiter une terre superbe placée dans le plus beau climat [...], à jouir de la meilleure santé, à respirer toujours l'air le plus pur et le plus salubre, à mener une vie libre, douce, tranquille, délivré de toutes les passions, de la jalousie même quoique environné de femmes charmantes, si ces femmes même peuvent encore faire partie du bonheur, je dis qu'il n'y a pas de peuple au monde plus heureux que la nation dont l'Ile de la Nouvelle Cythère est la patrie ${ }^{24}$.

24 Celui qui est «libre » est celui qui vit «environné de femmes charmantes ». Ensuite seulement, on envisage le complément qui étend ce bonheur à toute la société : tant mieux « si ces femmes » elles-mêmes peuvent être heureuses...

25 Dans la même logique qui se veut générale alors qu'on ne parle que d'une seule catégorie de sexe, Bougainville nous dit que l'amour règne en maitre car les filles ont de nombreux "amants»; mais aucune phrase ne vient parler des "amantes» d'un homme. Bref, pour les hommes, l'amour-libre tahitien se traduirait par le fait d'être polygame, ce qui maintient une certaine intégration dans (ce que les Français pensent être) la règle sociale locale. Pour les jeunes femmes non mariées, l'amour libre se traduirait par le fait d'entretenir de nombreuses liaisons avant le mariage ${ }^{25}$. Ainsi, des hommes venus d'Europe observent - croient observer - la manière dont les hommes polynésiens disposent des femmes et dont les femmes polynésiennes expriment ce que toutes les femmes du monde " désirent le plus».

26 Leur surinterprétation des attitudes féminines tahitiennes a persuadé les Français d'avoir rencontré une humanité encore « dans l'état de nature ». Pour les philosophes et naturalistes d'alors, la «nature » est le système du monde, tel qu'il fut donné à la Création (la vision courante est une sorte de déisme). De là découle un premier emploi, celui du qualificatif «naturels » pour désigner les peuples exotiques qui en sont restés 
plus ou moins au début (à condition qu'ils ne soient pas devenus trop noirs de peau). En effet, les Européens se voient vivre en "civilisation ", donc après l'apparition d'un contrat social (monarchique ou parlementaire, etc.) qui a permis de faire prospérer "les lois, les sciences et les arts». Les "sauvages» au contraire sont ceux qui vivent "sans lois ni arts" (ce furent les commentaires, du XVII ${ }^{e}$ au $\mathrm{XIX}^{\mathrm{e}}$ siècles, sur les Aborigènes d'Australie et sur les "Pécherais " du détroit de Magellan). Le terme de «naturels » permet d'éviter la connotation entièrement péjorative de "sauvages", de laisser un flou sur la différence entre "sauvages " et "barbares", de créer même le modèle du Bon Sauvage, et de dire que les sauvages véritables, ceux qui ne sont que des «brutes » (des animaux), sont ceux qui ont « dégénéré » pour raisons climatiques (et leur peau s'est assombrie!).

27 Un autre emploi dérivé concerne le corps. Le fonctionnement organique du corps est, comme celui des plantes, un domaine donné dès la Création et donc un domaine également "naturel». C'est pourquoi, on trouve à cette époque l'expression de "parties naturelles» pour désigner les organes sexuels. Or, pour le point de vue masculin de l'époque, la femme est plus «naturelle » que l'homme car le rôle féminin dans la communauté est envisagé surtout à partir de ses fonctions organiques spécifiques : mettre au monde et allaiter. De cette réduction de la femme à la fonction «naturelle » de l'enfantement et de l'allaitement découle une extension de la "nature » féminine à la sexualité et au désir. Chez la femme, le désir sexuel est plus "naturel » que chez l'homme. L'extension se poursuit avec l'idée masculine que «dans le beau sexe [...] [on trouve] cette tendresse, cette affection, ces sentiments vifs, plutôt fondés sur la passion que sur la raison.» Cette citation date de $1784^{26}$, mais l'opposition réservant la "raison » aux hommes et la "passion » aux femmes fut encore souvent invoquée par la suite, et nous savons que de nombreux propos contemporains n'y échappent toujours pas. Nous retrouvons ce point de vue chez Moerenhout (homme d'affaires et consul, installé à Tahiti au début du XIX $x^{e}$ siècle) dans sa description des femmes tahitiennes :

La toilette, quoique simple, était là, comme dans tous les pays du monde, une affaire de première importance, surtout pour les femmes, dont l'instinct, si la race humaine en possède un qui lui soit propre, est certainement de se parer et de chercher à plaire et, comme ailleurs encore, elles en parlaient longtemps d'avance. La danse, les fêtes passées ou à venir, étaient aussi des sujets fréquemment reproduits, avec d'autres non moins frivoles, mais qui, occupant toutes leurs pensées, pouvaient seuls les intéresser ${ }^{27}$.

28 Cette tendance naturelle (" instinctive ») des femmes en général - « chercher à plaire » - est encore plus visible chez les peuples «naturels", particulièrement ceux qui vont constamment «nus » en exhibant leurs " parties naturelles ». Nous voyons le cumul et la collision sémantiques qui ont été impliqués dans la surinterprétation française des mœurs tahitiennes.

Les femmes «blanches »

29 Quand on cherche à comprendre ce qui a constitué l'attirance des hommes européens d'alors pour les femmes polynésiennes, on oublie généralement de mentionner le schéma racial qui était alors installé dans la vision européenne de l'autre. Depuis longtemps, les géographes opposaient les peuples à peau noire, « vieille race de la zone torride " avait écrit Charles de Brosses en 1756, et ceux à peau «claire», ou du moins « cuivrée ». Le contraste entre « Nègres » et «Indiens » date du $\mathrm{XV}^{\mathrm{e}}-\mathrm{XVI}^{\mathrm{e}}$ siècle et 
connaîtra une grande application en Océanie avec l'invention européenne, au début du $\mathrm{XIX}^{\mathrm{e}}$ siècle, de l'opposition entre "Polynésiens » et « Mélanésiens » ${ }^{28}$.

Le lieutenant Caro écrivit, à propos des Tahitiens qui vinrent en pirogue au contact de l'expédition, qu'il y avait, en plus des adultes, «deux jeunes filles [...] un peu plus blanches que les hommes les plus blanchâtres ». On sait aujourd'hui que cela résultait d'une coutume qui consistait à empêcher les filles des familles de haut rang de demeurer longtemps à l'extérieur des maisons, afin qu'elles fussent aussi claires qu'à la naissance, en référence à une cosmologie solaire qui, de façon générale, valorisait la clarté.

31 Les raisons pour lesquelles l'apparence des jeunes Polynésiennes, surtout quand cellesci se dénudaient, a immédiatement captivé l'attention et le désir des arrivants sont donc multiples et il ne faut pas oublier l'obsession des Européens de l'époque sur la couleur de la peau. L'admiration pour la clarté de la peau des Polynésiennes est une mention omniprésente dans les récits de l'époque. Chacun s'extasiait. Fesche écrivit à propos de "Vénus » qui vint la première sur le navire : « elle était grande, bien faite et avait un teint que la plus grande partie des Espagnoles ne désavoueraient pas pour sa blancheur ». J. R. Forster (le naturaliste du deuxième voyage de Cook, 1773-1775) estima que Tahiti représentait «la plus belle variété » de l'espèce humaine exotique car la peau des Tahitiens était «moins basanée que celle d'un Espagnol, moins cuivrée que celle d'un Américain, plus claire que la peau la plus claire qu'on puisse trouver aux Indes orientales ». Le chirurgien Vivès mentionna dans son journal la présence du Tahitien Ahutoru qui avait accosté le premier jour, quand les vaisseaux de Bougainville louvoyaient encore :

[Il était] accompagné d'une fille ou femme de 16 à 18 ans, car la méprise est aisée à faire, paraissant très bien faite, ayant un morceau de drap blanc en pain de sucre autour de la tête et un autre à la ceinture, le reste du corps nu et aussi blanc qu'une européenne.

Une fois de retour en France, Vivès ajouta en 1774 :

notre imagination politiqua beaucoup dès cet instant, pour savoir si cette beauté n'était point étrangère au pays. Comment est-ce qu'un peuple aussi charmant pouvait être aussi éloigné d'Europe ? Et comment il se trouvait dans cette île aussi blanc, tandis que tout ce que nous avions vu dans les autres îles, depuis notre départ, n'y avait aucun rapport ? ${ }^{29}$

33 Vivès exprimait tout haut un contraste qui, sans aucun doute, a frappé tous les autres compagnons. Il est donc essentiel de traiter ensemble l'invention raciale de la Polynésie et l'invention sexiste-sexuelle de cette même Polynésie. Deux siècles de schémas raciaux furent précipités dans les jugements que les hommes Français portèrent sur les Tahitiennes dans ces quelques jours d'avril 1768 et qui, à leur tour, inaugurèrent le mythe de la sexualité polynésienne.

34 On relèvera aussi dans ce passage de Vivès un bel exemple de la tendance qui fit voir à ces voyageurs toute la société polynésienne sous le coup de l'excitation ressentie devant les jeunes filles nues. De la fille ou femme au corps «blanc », l'auteur passe au « peuple aussi charmant » qui est « aussi blanc».

Mauvais et Bons sauvages

Chez le bon sauvage, les femmes y sont comme Ève avant la Chute, libres d'exprimer leur désir "naturel». Le mauvais sauvage est donc celui dont les femmes n'exprimèrent pas spontanément leur désir devant l'arrivée d'hommes européens. Les 
Français ont aperçu ce sauvage avant d'entrer dans le Pacifique : dans le Détroit de Magellan. Ensuite ils l'ont vu dans les escales qui ont suivi Tahiti: les Samoans entr'aperçus par Bougainville ${ }^{30}$, puis, surtout, les peuples « noirs » des îles occidentales du Pacifique, qu'on appellera plus tard les « Mélanésiens »).

Tous les analystes des récits issus de l'escale tahitienne oublient que Tahiti fut nécessairement apprécié par contraste avec l'escale précédente. Or, cette escale était le Détroit. Pour les Français comme pour les Anglais, le schéma fut le même. Une fois partis des rives européennes puis américaines, l'inconnu et le danger commençaient en sortant de l'Atlantique, au moment de s'engager dans le Détroit. Là, les marins découvrirent des contrées dénudées, balayées par des vents glacés, des falaises sombres, des mers démontées. Les contacts établis à terre s'en ressentirent sans doute, car le jugement fut rapide et sans appel : on ne voyait que des sauvages miséreux, laids, vêtus de peaux de bêtes, qui n'avaient pas grand-chose à offrir qui soit digne de retenir l'attention des voyageurs. Nassau les décrivit ainsi : «[...] plus petits que les Français, d'une figure très désagréable, très maigres. Tous ont les dents gâtées ${ }^{31}$. Mais, une fois arrivés à Tahiti, les Français admirèrent la grande taille des insulaires et les dents qui leur parurent d'une blancheur éclatante. Quelques-uns des "sauvages " habitant la région du Détroit montèrent à bord. Mais, si les pirogues vinrent en portant des hommes et des femmes, seuls les premiers montèrent sur le bateau des Français ${ }^{32}$. À Tahiti, ce fut le contraire, du moins c'est ce que les Français crurent voir. On montra à ces habitants du Détroit divers objets, on leur donna de la nourriture. Mais Bougainville se plaignit de leur "puanteur insupportable ${ }^{33}$. À Tahiti, au contraire, les Français admirèrent la propreté corporelle et les huiles odoriférantes dont les corps étaient enduits. Les habitants du Détroit manifestèrent clairement leur incapacité de côtoyer la civilisation. Bougainville écrivit dans son livre :

Ils ne témoignèrent aucune surprise ni à la vue des navires, ni à celle des objets divers qu'on y offrit à leurs regards ${ }^{34}$; c'est sans doute que, pour être surpris de l'ouvrage des arts, il faut en avoir quelques idées élémentaires. Ces hommes bruts traitaient les chefs-d'œuvre de l'industrie humaine comme ils traitent les lois de la nature et ses phénomènes.

37 C'est-à-dire, pensent les Français, sans rien admirer ni comprendre. C'est pourquoi les Français ne se montreront pas trop sévères devant les «vols » d'un pistolet, d'une lunette, etc., opérés ensuite par les Tahitiens, car ces actes témoignaient au moins d'une capacité à admirer les « chefs d'œuvre de l'industrie humaine ».

Bougainville ajouta dans son commentaire conclusif :

Ces sauvages sont petits, vilains, maigres et d'une puanteur insupportable. Ils sont presque nus, n'ayant pour vêtement que de mauvaises peaux de loups marins [...] Leurs femmes sont hideuses et les hommes semblent avoir pour elles peu d'égards $[\ldots] .^{35}$

Dans son journal, il avait ajouté : « en vérité, lorsqu'on voit ces Sauvages, avec toute l'envie du monde de philosopher, on ne saurait préférer l'homme dans cet état de nature à l'homme civilisé ${ }^{36}$.

40 L'« état de nature » n'est donc pas toujours synonyme de l'Âge d'Or. Il faut encore que l'homme «naturel» ne paraisse pas misérable selon la classification européenne de l'époque. Il faut donc que le «naturel » vive dans une société ayant déjà élaboré un minimum d'organisation (des « chefs »). Il faut qu'il ait un corps « beau " (la référence d'alors est la statuaire antique), des dents bien «blanches » (la référence aux dents est une constante des descriptions de l'époque sur toutes les populations). Il faut aussi que 
l'homme «naturel» exprime par ses "coutumes» son admiration des «lois de la nature " - ce que les Tahitiens paraîtront avoir fait quand les Français auront l'impression que les Tahitiens ont érigé la sexualité en une fête publique et continuelle.

41 Il faut encore que les femmes du lieu ne paraissent pas trop "subjuguées " par les hommes. Rappelons quelques sous-entendus qui étaient évidents pour des intellectuels masculins de l'époque. La pulsion naturelle des femmes est gouvernée par l'Amour. La tendance sociale (ou naturelle) des hommes est le sentiment de propriété vis-à-vis des femmes. La liberté sexuelle des femmes traduit donc une faible subordination des femmes envers les hommes. Or, depuis le milieu du siècle, le degré de moindre subordination des femmes est considéré comme un signe de "civilisation" et d'éloignement de la «sauvagerie ». Qu'on ne s'y trompe pas! Il ne s'agit pas de l'indépendance sociale des femmes au sens moderne, mais uniquement de l'indépendance dont jouissent les femmes pour exprimer leur "nature ", c'est-à-dire leur position de mère et leur désir sexuel. À cette époque et pour longtemps encore, la femme par rapport à l'homme est classée au pôle de la "nature » par rapport à la " société ». Le modèle de la liberté avancée est la possibilité de choisir son époux ou son amant, le modèle de la «sauvagerie » étant la concubine esclave ou l'épouse « acquise par achat $»^{37}$.

42 Une culture tahitienne telle que Bougainville l'inventa, où les femmes - au moins celles non mariées - choisiraient elles-mêmes leurs amants et en changeraient constamment, était donc apte à représenter un stade avancé dans la marche vers la civilisation. Mais les descriptions des habitants vivant sur les îles que Dumont d'Urville appellera en 1831 les «îles noires " (Mélanésie) ne disaient rien de semblable et, de fait, quand Forster aborda avec Cook les côtes des archipels que le capitaine anglais baptisa « NouvellesHébrides» (aujourd'hui le Vanuatu) et "Nouvelle-Calédonie», il ne vit rien de semblable. D'une part, en vertu d'un schéma racial déjà installé, les voyageurs masculins européens avaient nettement moins tendance à admirer le corps des femmes noires rencontrées. D'autre part, il est vrai que, lors des premiers contacts, ils ne se sont pas vu offrir systématiquement des présentations sexuelles féminines. Ces observations, déjà orientées par le schème racial établi, vinrent le conforter en retour.

43 Commerson admirait chez les Tahitiens "l'honnêteté de leurs procédés envers les femmes qui ne sont nullement subjuguées chez eux comme chez les sauvages $»^{38}$. Mais Bougainville nota dans le Détroit que :

Leurs femmes sont hideuses et les hommes semblent avoir pour elles peu d'égards. Ce sont elles qui voguent dans les pirogues et qui prennent soin de les entretenir, au point d'aller à la nage, malgré le froid, vider l'eau qui peut y entrer [...] ; à terre, elles ramassent le bois et les coquillages, sans que les hommes prennent aucune part au travail. Les femmes mêmes qui ont des enfants à la mamelle ne sont pas exemptes de ces corvées. Elles portent sur le dos les enfants pliés dans la peau qui leur sert de vêtement. ${ }^{39}$

Bien entendu, des femmes « hideuses» ne sauraient vivre dans une société de liberté. On voit ainsi comment le jugement sur la liberté des femmes tahitiennes fut le résultat de deux projections du regard européen masculin. La mesure de l'égalité sociale était faite par rapport à la liberté donnée aux femmes d'offrir leur corps, mais aussi par rapport à la beauté qui semblait se dégager de leur corps, cette impression étant ellemême le résultat des contrastes raciaux déjà installés : la "blancheur " inattendue de ces « Indiennes ».

Dans son livre de 1778, Forster écrivit à propos des jeunes filles de Tahiti : 
Nous sommes certains maintenant qu'on ne peut guère trouver un autre pays où les jeunes femmes célibataires posséderaient autant qu'à Tahiti et les îles voisines toute latitude pour accueillir diverses sortes de jeunes hommes et pour s'abandonner dans leurs bras. ${ }^{40}$

Or, ce passage sur les femmes tahitiennes se trouve dans un développement de Forster sur le contraste, à ses yeux d'Européen, entre les femmes qu'il a observées aux Nouvelles-Hébrides et en Nouvelle-Calédonie - donc dans la région qui sera bientôt nommée "Mélanésie» - et celles de Tahiti et des autres îles polynésiennes (la généralisation se fait immédiatement). Les premières sont caractérisées d'emblée de la façon suivante : «elles portent les fardeaux, elles font tout le travail domestique»; leurs maris se comportent envers elles avec « oppression et grossièreté ». Elles «sont habituées à se soumettre ». Au contraire, à Tahiti, mais aussi à Tonga et aux Marquises :

Sur les îles de Tahiti, de la Société, des Amis [Tonga] et des Marquises, le beau sexe est déjà haussé vers une plus grande égalité avec les hommes; et s'il n'y avait pas d'autre raison, celle-là suffirait à nous permettre de conclure que ces insulaires ont émergé du stade de la sauvagerie et doivent être rangés à un niveau juste au dessus du stade de la barbarie. ${ }^{41}$

Le malentendu fut immense : des hommes occidentaux, pour qui c'était la femme en général et non l'homme qui représentait l'état de nature et l'instinct de l'amour, débarquèrent dans une Polynésie où ce qu'ils prirent à tort pour un appel général des femmes à l'amour leur fit voir tout ce qu'ils observaient à travers le prisme de leur conception du féminin ${ }^{42}$.

\section{BIBLIOGRAPHIE}

BEAGLEHOLE J.-C. (dir.), 1955-1974, The Journals of James Cook, Cambridge, Cambridge University Press for The Hakluyt Society.

BLOCH Maurice et BLOCH, Jean-H., 1980, « Women and the dialectics of nature in eighteenthcentury French thought », in Carol P. MacCormack et Marilyn Strathern (dir.), Nature, culture and gender, Cambridge, Cambrige University Press, p. 25-41.

BOUGAINVILLE Louis-Antoine (Comte de), 1966, Voyage autour du monde, Paris, Union Générale d'Editions (coll. 10/18) (éd. originale : 1771, Voyage autour du monde par la frégate La Boudeuse et la flûte L'Etoile... 1766, 1767, 1768, 1769, Paris, Saillant et Nyon).

,-- 1968 , «Journal de Bougainville » (retranscrit par Etienne Taillemite pour l'ensemble du séjour à Tahiti, in extenso, à l'exception des indications de sondage), dans le numéro spécial, E. Taillemite (dir.), « Hommage à Bougainville », Journal de la société des océanistes, 1968, n 24, p. 11-34.

COMMERSON Philibert, 1968, Extrait d'une description générale des coutumes de « l'isle de la Nouvelle Cythère ", synthèse manuscrite ajoutée en « Post-scriptum » au journal attribué par E. Taillemite à Pierre Duclos-Guyot ", Journal de la société des océanistes, 1968, n² 24, p. 41-48.

DANIELSSON Bengt, 1981, Tahiti autrefois, Papeete, Hibiscus Editions. 
FESCHE Felix, 1968, « Journal de Fesche », Journal de la société des océanistes, 1968, n²4, les notes des p. 11-34.

FORSTER Johann Reinhold, 1778, Observations made during a voyage round the world, in Nicolas Thomas, Harriet Guest et Michael Dettelbach (dir.), 1996, Observations made during a voyage round the world, Johann Reinhold Forster, Honolulu, University of Hawai'i Press (le texte original de 1778 de Forster, paru à Londres chez G. Robinson, est entièrement reproduit).

JOLLY Margaret, 1992, "'Ill-natured comparisons' : racism and relativism in European representations of ni-Vanuata from Cook's second voyage », History and Anthropology, 5 (3-4), p. 331-364.

LAPÉROUSE Jean-François Galaud de, 1985, in John Dunmore et Maurice de Brossard (dir.), Le voyage de Lapérouse, 1785-1788 : récit et documents originaux, Paris, Imprimerie Nationale.

MARTIN-ALLANIC Jean-Etienne, 1964, Bougainville navigateur et les découvertes de son temps, Paris, Presses Universitaires de France.

NASSAU Prince de, 1968, « Journal du prince de Nassau-Siegen », Journal de la société des océanistes, $1968, n^{\circ} 24$, p. $49-54$.

OLIVER Douglas, 1974, Ancient Tahitian Society, Honolulu / Canberra, University of Hawaii Press / Australian National University Press.

ORANS Martin, 1996, Not even wrong: Margaret Mead, Derek Freeman and the Samoans. Novato (Cal.), Chandler \& Sharp.

TAILLEMITE Etienne (dir.), 1977, Bougainville et ses compagnons autour du monde 1766-1769, journaux de navigation établis et commentés par, Paris, Imprimerie Nationale.

TCHERKEZOFF Serge, 1993, « Une hypothèse sur la valeur du 'prix de la fiancée' nyamwezi », in Françoise Héritier et Elizabeth Copet-Rougier (dir.), Les Complexités de l'alliance. Economie, politique et fondements symboliques de l'alliance. Volume 3 : Afrique, Paris, Editions des archives contemporaines (coll. « ordres sociaux »), p. 51-80.

--, 2001a, Le mythe occidental de la sexualité polynésienne (1928-1999) : Margaret Mead, Derek Freeman et Samoa, Paris, Presses Universitaires de France.

--, 2001b, « Is anthropology about individual agency or culture ? Or why 'Old Derek' is doubly wrong ; Samoa again : on 'Durkheimian bees', Freemanian passions and Fa'amu's 'confession' », Journal of the Polynesian Society, Journal of the Polynesian Society, 110 (1) pp. 59-78; 110 (4), pp. 431-436.

--, 2002, « L'humain et le divin : quand les Polynésiens ont découvert les explorateurs européens au XVIII ${ }^{\mathrm{e}}$ siècle ", Ethnologies comparées, 5 (pages électroniques : <http://alor.univ-montp3.fr/ cerce/revue.htm>)

--, 2003a, FaaSamoa, une identité polynésienne (économie, politique, sexualité) : l'anthropologie comme dialogue culturel. Paris, L'Harmattan (« Connaissance des Hommes»).

--, 2003b, « A long and unfortunate voyage toward the invention of the Melanesia / Polynesia opposition (1595-1832)», The Journal of Pacific History (special issue : « Dumont d'Urville's Oceanic Provinces : Fundamental Divisions or Intangible Invention », Geoffrey Clark, dir), 38 (2), p. 175-196.

--, 2004a, Tahiti, 1768 : la face cachée des premiers contacts et la naissance du mythe occidental, Pape'ete, Au Vent des îles. 
,$-- 2004 b$, 'First Contacts' in Polynesia : the Samoan case (1722-1848). Western misunderstandings about sexuality and divinity, Canberra / Cristchurch, Journal of Pacific History Monographs / Macmillan Brown Centre for Pacific Studies.

--, 2005, «Le désir féminin, l'anatomie de Priape et l'ardeur du French lover : stéréotypes de la sexualité chez les voyageurs français lors des premiers contacts en Polynésie », in Véronique Fillol (dir.), Actes du $17^{\mathrm{e}}$ Colloque C.O.R A.I.L., Lieux communs et représentations en Océanie (Nouméa, décembre 2004), Nouméa, Grain de Sable.

--, n.d., « A reconsideration of the role of Polynesian women in the early encounters with Europeans : Supplement to Marshall Sahlins' Voyage Around the Islands of History ", in Margaret Jolly, Serge Tcherkezoff, Darrell Tryon (eds), Oceanic Encounters, Honolulu, University of Hawaii Press.

VOLTAIRE, 1987, Correspondance (établie par Théodore Besterman), Paris, Gallimard (« La Pléiade »), vol. XI, p. 702-703, lettre n 13.770.

\section{NOTES}

1. Bougainville $1966: 194-95$.

2. Ils relatent comment ils communiquaient par signes.

3. Voir Tcherkezoff 2004a : chap. 5.

4. Voir les citations dans Tcherkezoff n.d.

5. Nassau $1968: 50$.

6. Commerson $1968: 43$.

7. Auquel on n'a pu avoir accès sous forme publiée que dans les années 1960, grâce au travail d'un historien néo-zélandais : voir J.C. Beaglehole (ed.) 1955-1963. Pour l'étude des déformations, voir Tcherkezoff 2004a : chap. 8-9.

8. Voir Tcherkezoff 2004a : 278 .

9. Voir Tcherkezoff 2004a: 276 .

10. Voir Tcherkezoff 2004a : chap. 9.

11. Voltaire ([1774] 1987 : 702.

12. Les arguments sur la possibilité de cette généralisation et les citations in extenso sont présentés dans deux ouvrages qui analysent en détail le cas tahitien et le cas samoan, en ajoutant des exemples marquisisens, tongiens, maoris (Tcherkezoff 2004a, 2004b) et dans un article (n.d.) qui compare les visions française et anglaise des « premiers contacts » en Polynésie.

13. Fesche $1968: 30$.

14. Fesche $1968: 15-16$.

15. Nassau $1968: 50$, Martin-Allanic $1964: 665$.

16. Lapérouse 1985 : II, 477.

17. Pour l'interprétation polynésienne de la nature des Européens, voir Tcherkezoff 2002 et, pour une version élargie (mais en anglais) : 2004a : chap. 9. Pour les théories de la conception et l'hypothèse relative à l'âge des filles présentées, voir Tcherkezoff 2003a : 372-383 ; 2004a : 405-454, 495-509.

18. Lapérouse 1985 : II, 477.

19. Lettre de Bachaumont, datée du 11 juillet 1769 (Martin-Allanic 1964 : 970). 
20. Dans le cas de Mead à Samoa, on dispose même de ses notes de terrain (en partie publiées : Orans 1996) qu'on peut confronter au texte publié (Tcherkezoff 2001a, 2001b, 2003a). Pour les analyses de Handy, voir Tcherkezoff 2004a : 474-486.

21. Bougainville $1966: 185$.

22. Fesche $1968: 12$.

23. Commerson $1968: 41-48$.

24. Fesche in Taillemite ed. $1977: 88$.

25. Bougainville $1966: 215-216$.

26. Citation de LaMettrie, dans la préface de son Système physique et moral de la femme, 1784 , que nous empruntons à Bloch et Bloch, $1980: 34$.

27. Cité par Danielsson 1981 : 372.

28. Tcherkezoff $2003 b$.

29. Voir Taillemite ed. 1977 : I, 30, II, 235.

30. Après Tahiti, Bougainville passe devant les Samoa. " Je ne crois pas ces hommes aussi doux que les Tahitiens : leur physionomie était plus sauvage [...]» (Bougainville 1966 : 238 ; voir le récit complet dans Tcherkezoff 2004b : chap. 2).

31. « Journal de Nassau » cité par Martin-Allanic $1964: 616$.

32. Cité par Martin-Allanic $1964: 622$.

33. Dans le livre (Bougainville 1966 : 155) et dans le journal (cité par Martin-Allanic $1964: 622-23)$.

34. Bougainville paraît oublier que ces peuples ont vu passer et même accoster des navires européens depuis 1520.

35. Bougainville $1966: 155$.

36. Martin-Allanic $1964: 623$.

37. Autre grand sujet de malentendu chez les Occidentaux que le mariage africain « par achat », malentendu qui va durer jusqu'au milieu du $\mathrm{xx}^{\mathrm{e}}$ siècle : voir Tcherkezoff 1993.

38. Commerson $1968: 41-48$.

39. Bougainville $1966: 155-6$.

40. Cité in Oliver 1974 : 357 (notre trad.).

41. Ce passage de 1778 de J. R. Forster est cité par Margaret Jolly (1992 : 334) (notre trad.).

42. Et de leur conception de la sexualité masculine, celle des Polynésiens (qui seraient constamment obnubilés par le sexe féminin) et la leur (la « réputation » de la "bouillante ardeur française », la générosité de Priape pour les Français, etc.) : voir Tcherkezoff 2005.

\section{RÉSUMÉS}

Dans une première partie, nous citerons les écrits qui racontent les "premiers contacts " entre Français et Polynésiens $(1768,1787)$. On y voit de fortes contradictions entre les conclusions généralisantes publiées, portant sur la liberté sexuelle pré maritale, dans un culte constant de l'amour pratiqué en public, et certains faits pourtant évoqués dans les journaux de bord: des 
présentations sexuelles forcées de très jeunes filles apeurées. Dans la deuxième partie, nous tenterons de comprendre cet aveuglement : il est dû à la vision européenne masculine d'alors sur la nature des femmes, et à un schéma racial déjà installé qui octroya par avance aux Polynésiennes la beauté de femmes « presque blanches ».

In the first part, are presented the European narratives of «first contacts " with Polynesians (Tahiti 1768, Samoa 1787). There are strong contradictions between the concluding and generalising views held by the visitors about the «customs » of the people encountered and some factual descriptions which are to be found in unpublished journals and which evoke very young and frightened girls, forcefully presented for a sexual act. In the second part, a question is raised : how European visitors could have made their conclusions about «free-love " in spite of this context? The answer lies within the European masculine view of the time about the "nature » of female " desire ", as well as within the European view on racial hierarchy among the "savages", which made the Polynesian women appear attractive because their skin was «nearly white ».

\section{INDEX}

Mots-clés : Bougainville, femmes, nature, sexualité, utopie Index géographique : Polynésie

\section{AUTEUR}

\section{SERGE TCHERKEZOFF}

Serge TCHERKEZOFF est directeur d'études à l'EHESS. Il dirige le Centre de recherches et de documentation sur l'Océanie (UMR CNRS, EHESS, U. de Provence). Il a édité avec Fr. DouaireMarsaudon, Le Pacifique-sud aujourd'hui, identités et transformations (CNRS Ed. 1997) et publié sur la société contemporaine de Samoa et les malentendus entre Polynésiens et Occidentaux dans les domaines de l'économie, de la politique et des rapports de sexe (Le mythe de la sexualité polynésienne, P.U.F. 2001 ; FaaSamoa, L'Harmattan 2003), ainsi que sur l'ethno-histoire des premières rencontres entre Polynésiens et Européens (Tahiti 1768, Au Vent des Iles, 2004 ; First Contacts : the Samoan Case, 1722-1840, Australian National University, 2004). Il a publié auparavant un ouvrage sur les formes de pouvoir sacré et sur la logique des classifications dualistes, principalement avec des exemples africains (Le Roi Nyamwezi, la droite et la gauche, MSH, 1983 ; Dual Classification Reconsidered, Cambridge, 1987), ainsi qu'une série d'articles sur l'histoire et sur l'épistémologie des modèles anthropologiques. 